\title{
Sheep meat Beni-Guil breed: A local meat labeled PGI to promote quality and preserve biodiversity in Morocco
}

\author{
Kamal Belhaj ${ }^{1, *}$, Farid Mansouri ${ }^{1}$, Melhaoui Reda ${ }^{1}$, Yassine Taaifi ${ }^{1}$, Youssef Rbah $^{1}$, Marianne Sindic ${ }^{2}$, Mohamed \\ Boukharta $^{3}$, Hana Serghin-Caid ${ }^{1}$, and Ahmed Elamrani ${ }^{1}$ \\ ${ }^{1}$ Laboratory of Agricultural Production Improvement, Biotechnology and Environment, Faculty of Sciences, University Mohammed \\ First, 717, 60000 Oujda, Morocco. \\ ${ }^{2}$ Analysis Quality and Risk Unit, Laboratory of Food Quality and Safety (QSPA), Gembloux Agro-Bio Tech, University of Liège, 5030 \\ Gembloux, Belgium \\ ${ }^{3}$ Institute of Agricultural Industries, High school of Charlemagne, 4500 Huy, Belgium.
}

\begin{abstract}
Currently, meat consumers are more conscious about the authentic meat products linked to their origin, which generates a growing demand for labeled sheep meat. In eastern Morocco, the Beni-Guil (BG) sheep meat is a Protected Geographical Indication (PGI) product. This study aimed to assess the variation of intramuscular fat quality of BG sheep in its cradles $(\mathrm{Cr})$. The chemical composition of the longissimus lumborum muscle was evaluated from 20 female lambs obtained in the two main cradles of the BG breed (Ain Beni-Mathar (BG-Cr1) and Tendrara (BG-Cr2). The results show that the FA profile was affected by $\mathrm{Cr}$ $(p<0.05)$. The oleic acid, palmitic acid, stearic acid, and linoleic acid are the dominants FA of analyzed meats. The polyunsaturated fatty acids (PUFA)/saturated fatty acids (SFA) and PUFA n-6/PUFAn-3 ratios respectively range from 1.15 and 5.02 for BG-sheep meats from $\mathrm{Cr} 1$ to 1.36 and 10.73 for those from $\mathrm{Cr} 2$. Overall, the results indicate that the analyzed meats have an interesting FAs profile with favorable ratios and indices. Thus, this study provides new information on the lipid quality of BG-IGP sheep meat produced extensively in two main cradles of this typical Moroccan sheep breed.
\end{abstract}

\section{Introduction}

The global demand for safe and high-quality meat has increased significantly in recent years. Consumers attach great importance to the extrinsic meat quality in response to raising safety and health concerns, and consequently, are more conscious about the authentic meat products linked to their origin. This circumstance generates a growing demand for labeled sheep meat. The provenance of meat is essential information for breeders, retailers, and consumers. For the latter, red meat may have a particular value associated with the geographical origin or production system from which they derive, e.g., "Protected Designation of Origin (PDO)", "Protected Geographical Indication (PGI)", and "organic", etc.

The use of local sheep breeds, reared under traditional-local systems and commercialized under the labels of designation of origin, has become very important in recent decades. In Morocco, sheep production is based mainly on pastoral systems with pure local breeds, i.e., Beni-Guil, Timahdite, Sardi, D'man, and Boujaad. The Beni-Guil sheep breed is considered the typical sheep of eastern Morocco and is recognized as the first red meat to be regarded as a PGI in Morocco. This sheep breed is born, reared, and slaughtered in the eastern Moroccan region, fed a particular diet, and reared under a specific pastoraltranshumant system. Consequently, it has a unique sensory and nutritional quality meat linked to this distinct environment and natural grazing system. BeniGuil sheep meat is one of the most consumed meat due to its sensory, organoleptic, and attractive nutritional value [1].

These characteristics imply its potential use in a sustainable and/or biological economic system based on the traditional transhumant grazing system. The meat quality is affected by intrinsic and extrinsic factors, which are generally related to a geographical area. Previous studies have reported geographical area (dietary differences between regions) on fatty acids profile and sensory quality [2]. Notably, the flavor is associated with the types of food, where lambs raised under the pasture-based system produce meat with pastoral flavor.

This paper aims to study the variation of intramuscular fat quality of PGI-Beni-Guil local sheep breeds according to in its main cradles. To date, no available studies have looked at the effects of the cradle on the meat quality characteristics of the Beni-Guil sheep breed. This work is a continuation of the one started in 2016-2017 to confirm the effect of the cradle on the Beni-Guil meat quality [3].

\footnotetext{
${ }^{*}$ Corresponding author: belhaj.kama190@gmail.com
} 


\section{Material and methods}

\subsection{Animals}

Beni-Guil sheep are distributed over the eastern region of Morocco. It is well suited to the harsh climate and poor pasture conditions and is classified as a meat production breed. It is a breed characterized by fire brown head, ears, prothorax, and legs without white spots; white fleece; absence of horns in the female (Fig. 1). The rams usually have coarse and longhorns that are pale and spiral. Animals were reared under transhumant-local production systems in the pasture of eastern Morocco highlands. This study was done on twenty-four 7-month-old of Beni-Guil female lambs weaned at the age of 3 months. The samples were taken from the two main cradles of the Beni-Guil breed: Female lambs of the Beni-Guil breed reared and slaughtered in Ain Beni-Mathar $(\mathrm{Cr} 1 ; \mathrm{n}=12)$ and Tendrara $(\mathrm{Cr} 2 ; \mathrm{n}=12)$. The pre-determined live weight of studied animals was $35.67 \mathrm{~kg}$ for $\mathrm{Cr} 1$ and $34.48 \mathrm{~kg}$ for $\mathrm{Cr} 2$. Female lambs were slaughtered in their areas of origin in the slaughterhouse.

Further details about rearing, diet composition, and slaughter conditions are described by [4]. The sampling was carried out in spring 2018. The longissimus lumborum samples were taken 24 hours post-mortem with the assistance of a slaughterhouse veterinarian.
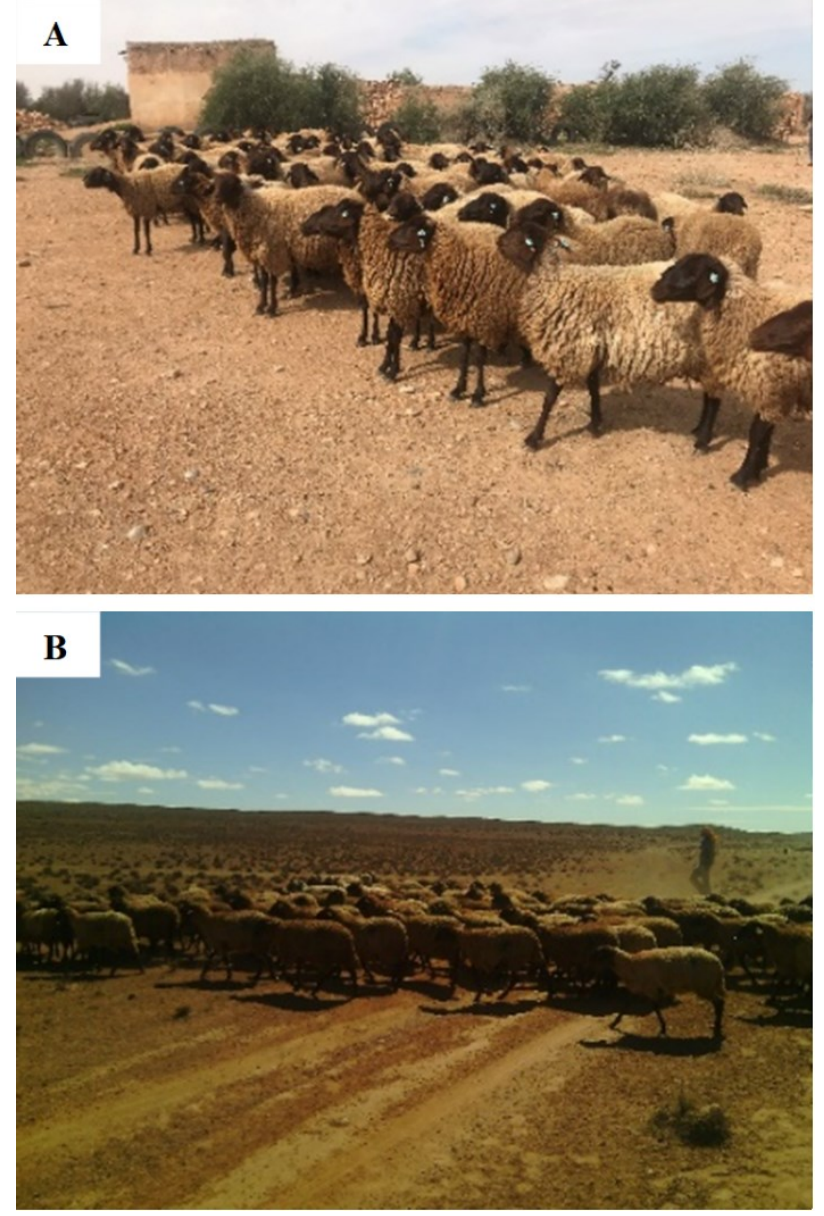

Fig 1. Beni-Guil female lambs (18 months old) in Ain BeniMathar region (A) and Tendrara region (B)

\subsection{Fatty acids composition}

The fat was extracted according to the Bligh Dyer method [5]. The fatty acids were methylated, and the samples were analyzed by Gas Chromatography equipped with a flame-ionization detection and capillary column. The nonadecanoic acid $1 \mathrm{mg} / \mathrm{ml}$ was used as an internal standard to calculate the fatty acid concentration.

\subsection{Statistical Analyses}

All the data were presented as means \pm standard deviation of triplicate determinations. The statistical analyses were achieved using the software SPSS version 20 and considering the cradle (Ain Beni-Mathar cradle vs. Tendrera cradle) as the source of variation. The means were compared using the $t$-test, and the significance was declared at $p<0.05$.

\section{Results and discussion}

The prevalent fatty acid content, ratios, and lipid health indices of the Beni-Guil sheep meat are shown in Table 1. The results show that the cradle has a significant effect on the fat quality of BG-PGI sheep meat $(p<0.05)$. The results show no significant differences between the studied groups for the dominant saturated fatty acids (Palmitic: 2507,03 vs. 2652,14 and stearic acid: 1381,26 vs. $1426,97 \mathrm{mg} / \mathrm{kg}$ of muscle, respectively for $\mathrm{Cr} 1$ and $\mathrm{Cr} 2$ ) and Monounsaturated fatty acid (oleic acid: $3735,01 \mathrm{vs} .3707,43 \mathrm{mg} / \mathrm{kg}$ of muscle).

However, female lambs from $\mathrm{Cr} 2$ showed significantly higher value for polyunsaturated fatty acids than $\mathrm{Cr} 1(p \leq 0.001)$. On the other hand, no significant difference between $\mathrm{Cr} 1$ vs. $\mathrm{Cr} 2$ was found for the SFA and MUFA sums. Concerning lipid health ratios and indices, the meat from $\mathrm{Cr} 2$ represents the better ratio (PUFA/SFA: 0.93 vs. 0.83) and indices (Thrombogenic: 0.93 vs. 0.83 and Atherogenic index: 0.82 vs. 0.69 ). In contrast, the meat of $\mathrm{Cr} 1$ has a better PUFA-n6/PUFAn-3 ratio (5.02 vs. 10.73). In the present study, oleic (C18:1), palmitic (C16:0), stearic (C18:0), and linoleic acid (C18:2n-6) represent more than $80 \%$ of the total fatty acid concentration of the longissimus lumborum of Beni-Guil sheep meat.

The dominant FA in the obtained profile was C18:1 in the meat of two studied groups, followed by $\mathrm{C} 16: 0$ and C18:0 (Table 1). These results align with those reported by several authors for sheepmeat $[4,6]$. To fight against certain diseases linked to the current lifestyle, the ratio of PUFA/SFA recommended should be greater than 0.4 and that of n-6/n-3 between 1 and 4 . These two ratios are used as a common index to assess lipid nutritional quality. The omega-6/omega-3 ratio plays an essential role, in particular in the prevention of cardiovascular diseases [7]. The results of this study show that the cradle affects these ratios significantly. The muscle of BG-Cr2 contains more PUFA resulting in the higher PUFA/SFA ratio (1.15 vs. 1.36$)$, while the BG-Cr1 one shows a lower n-6/n-3 (5.02 vs. 10.73). The 
individual effect of fatty acid on cholesterol metabolism was evaluated by calculating the Hypocholesterolemic (h)/Hypercholesteremic (H) ratio. The results demonstrate that the meat of female lambs reared in $\mathrm{Cr} 2$ has the better $\mathrm{h} / \mathrm{H}$ ratio ( 1.72 vs 2 ).

This finding could be explained by the breeding system and the pasture availability and diversity between studied cradles. In the $\mathrm{Cr} 2$, the breeding system is more extensive than $\mathrm{Cr} 1$, where the Artemisia herbaalba and Stipa tenacissima formations are in an advanced degradation state compared to $\mathrm{Cr} 2$ [8]. Consequently, the farmers of $\mathrm{Cr} 1$ adopt a semiextensive system using barley and Lucerne hay as a food supplementation to satisfy the different needs of their herds. The wormwood and alfa species are richer in bioactive substances such as antimicrobials and antioxidants, which lead to the decrease in ruminal biohydrogenation and PUFA degradation [9]. As a result, the production of meat rich in PUFA is the case of meat from $\mathrm{Cr} 2$. Concerning the PUFA-6/PUFA-3 ratio, the recorded results are higher than the recommended value. The incorporation of alfalfa could explain the lower value recorded in meat produced in $\mathrm{Cr} 1$ compared to $\mathrm{Cr} 2$ in the food ratio rich in n-3 fatty acids.

Table 1. Fatty acids content of Beni-Guil sheep meat in Ain Beni-Mathar and Tendrara region

\begin{tabular}{|c|c|c|c|}
\hline & \multicolumn{2}{|c|}{ Cradle } & \multirow[b]{2}{*}{$\begin{array}{c}\text { P- } \\
\text { value }\end{array}$} \\
\hline $\begin{array}{c}\text { Prevalent FAs } \\
\left(\text { mgkg }^{-1} \text { of muscle), }\right. \\
\text { sums, ratios, } \\
\text { and indices }\end{array}$ & BG-Cr1 & BG-Cr2 & \\
\hline Oleic acid & $3735,01 \pm 660,40$ & $3707,43 \pm 517,26$ & 0.580 \\
\hline Palmitic acid & $2507,03 \pm 436,01$ & $2652,14 \pm 201,68$ & 0.903 \\
\hline Stearic acid & $1381,26 \pm 308,77$ & $1426,97 \pm 151,74$ & 0.289 \\
\hline linoleic acid & $737,62 \pm 230,91$ & $1235,60 \pm 191,82$ & $<0.001$ \\
\hline Arachidonic acid & $208,36 \pm 98,53$ & $697,24 \pm 280,83$ & $<0.001$ \\
\hline Linolenic acid & $90,15 \pm 24,10$ & $116,26 \pm 47,99$ & 0.1 \\
\hline $\begin{array}{c}\text { Docosahexaenoic } \\
\text { Acid }\end{array}$ & $83,24 \pm 17,73$ & $76,21 \pm 16,58$ & 0.278 \\
\hline SFA & $4658,38 \pm 732,67$ & $4978,27 \pm 338,75$ & 0.167 \\
\hline MUFA & $4064,64 \pm 704,35$ & $4192,55 \pm 413,29$ & 0.572 \\
\hline PUFA & $1286,84 \pm 394,74$ & $2552,02 \pm 520,80$ & $<0.001$ \\
\hline UFA & $5351,49 \pm 822,38$ & $6744,57 \pm 613,95$ & $<0.001$ \\
\hline PUFA/SFA & $1,15 \pm 0,078$ & $1,36 \pm 0,08$ & $<0.001$ \\
\hline$\omega-6 / \omega-3$ & $5.02 \pm 1.74^{\mathrm{a}}$ & $10.73 \pm 4.38^{\mathrm{b}}$ & 0.001 \\
\hline $\mathrm{TI}$ & $0.93 \pm 0.11^{\mathrm{c}}$ & $0.83 \pm 0.04^{b}$ & 0.002 \\
\hline AI & $0.82 \pm 0.15^{\mathrm{c}}$ & $0.69 \pm 0.01^{\mathrm{cb}}$ & 0.002 \\
\hline $\mathrm{h} / \mathrm{H}$ & $1.72 \pm 0.26^{\mathrm{a}}$ & $2 \pm 0.06^{\mathrm{b}}$ & $<0.001$ \\
\hline \multicolumn{4}{|c|}{ 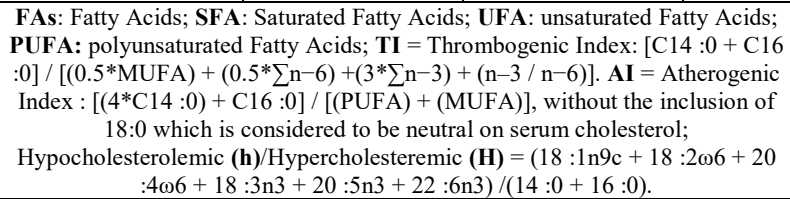 } \\
\hline
\end{tabular}

\section{Conclusion}

The present research allowed us to study the variation of sheep meat quality from the "Beni-Guil-PGI breed" in its main cradles. The results show that the lipid nutritional value of this labeled breed is not homogeneous. To increase consumers' loyalty, the PGI label should consider the cradle effect on meat quality in their regulatory directive to provide a standard product from one purchase to another. Further investigations are needed to study the cradle effects on meat traits, evaluating, in particular, the sensorial quality to identify the olfactory biomarkers for each cradle.

\section{References}

1. K. Belhaj, F. Mansouri, M. Sindic, M.-L. Fauconnier, M. Boukharta, H. Serghini Caid, and A. Elamrani, The Scientific World Journal, 2021, (2021)

2. S.W. Erasmus, M. Muller, and L.C. Hoffman, Journal of the Science of Food and Agriculture, 97, 19791996 (2017)

3. K. Belhaj, F. Mansouri, A. Benmoumen, M. Sindic, M.-L. Fauconnier, M. Boukharta, C.H. Serghini, and A. Elamrani, Archives Animal Breeding, 63, 471$482(2020)$

4. K. Belhaj, F. Mansouri, M. Sindic, M.-L. Fauconnier, M. Boukharta, H. Serghini Caid, and A. Elamrani, Journal of Food Quality, 2021, (2021)

5. E.G. Bligh and W.J. Dyer, Canadian journal of biochemistry and physiology, 37, 911-917 (1959)

6. M. Blasco, M.M. Campo, J. Balado, and C. Sañudo, Journal of the Science of Food and Agriculture, 99, 3335-3342 (2019)

7. NL. Howes, AEDA Bekhit, D.J. Burritt, and A.W. Campbell, Comprehensive Reviews in Food Science and Food Safety, 14, 22-36 (2015)

8. A. Bechchari, A. El Aich, H. Mahyou, B. Baghdad, and M. Bendaou, Journal of Materials and Environmental Science, 5, 2572-2583 (2014)

9. V. Vasta, D. Aouadi, D.M. Brogna, M. Scerra, G. Luciano, A. Priolo, and H.B. Salem, Meat science, 95, 235-241 (2013) 